\title{
The utility of pleural amylase measurement in metastatic mucinous adenocarcinoma
}

\author{
${ }^{1}$ A Fahim, ${ }^{2} \mathrm{JA}$ Kastelik \\ ${ }^{1}$ Specialist Registrar, Respiratory Medicine; ${ }^{2}$ Consultant Respiratory Physician, Castle Hill Hospital, Cottingham, UK
}

\begin{abstract}
We describe a case of exudative pleural effusion in a patient with mucinous pulmonary adenocarcinoma. Pleural fluid examination showed markedly raised amylase with normal serum amylase concentration. There was no clinical or radiological evidence of oesophageal rupture or pancreatitis. The case illustrates the importance of considering pulmonary malignancy in the context of amylaserich pleural effusion. Causes of amylase-rich pleural effusion and the significance of isoenzyme analysis are discussed.
\end{abstract}

Correspondence to A Fahim, Castle Hill Hospital, Cottingham HUI6 5JQ, UK

tel. $+44(0) 1482624067$ e-mail ahmedfahim@doctors.org.uk

KEYWORDS Amylase isoenzyme, pleural effusion, pulmonary adenocarcinoma

DECLARATION OF INTERESTS No conflict of interests declared.

\section{CASE REPORT}

A 73-year-old woman with a background of ChurgStrauss vasculitis, mononeuritis multiplex and hypertension presented with progressive dyspnoea and worsening exercise tolerance. There was no history of vomiting or chest pain. She was an ex-smoker with a 20 pack-year history of smoking. There was no history of excess alcohol intake. Her regular medications included prednisolone and azathioprine for vasculitis.

On examination, the patient was haemodynamically stable. A respiratory system examination was consistent with a right-sided pleural effusion. A systemic examination, including a breast examination, was unremarkable. Her chest radiograph (Figure I) confirmed moderate right pleural effusion, therefore an intercostal drain was inserted. Thoracic computed tomography (CT) did not show any evidence of intrapulmonary mass or mediastinal lymphadenopathy. In addition, there was no evidence of oesophageal rupture. An abdominal CT scan was normal without any evidence of pancreatic malignancy or pancreatitis. Pleural fluid analysis revealed exudative pleural effusion with markedly raised amylase of $I, 890$ units/litre $(u / l)$, with a pleural fluid to serum amylase ratio of 36 . Serum and urinary amylase were $53 \mathrm{u} / \mathrm{l}$ and $483 \mathrm{u} / \mathrm{l}$ respectively. However, an isoenzyme analysis on pleural or serum amylase was not carried out in this case. The pleural fluid cytology was negative for malignancy.

Pleural biopsy revealed the presence of metastatic mucinous adenocarcinoma. Immunohistochemistry showed it to be positive for thyroid transcription factor-I (TTF-I) and carcinoembryonic antigen (CEA), suggesting a pulmonary origin for the malignancy. The patient was

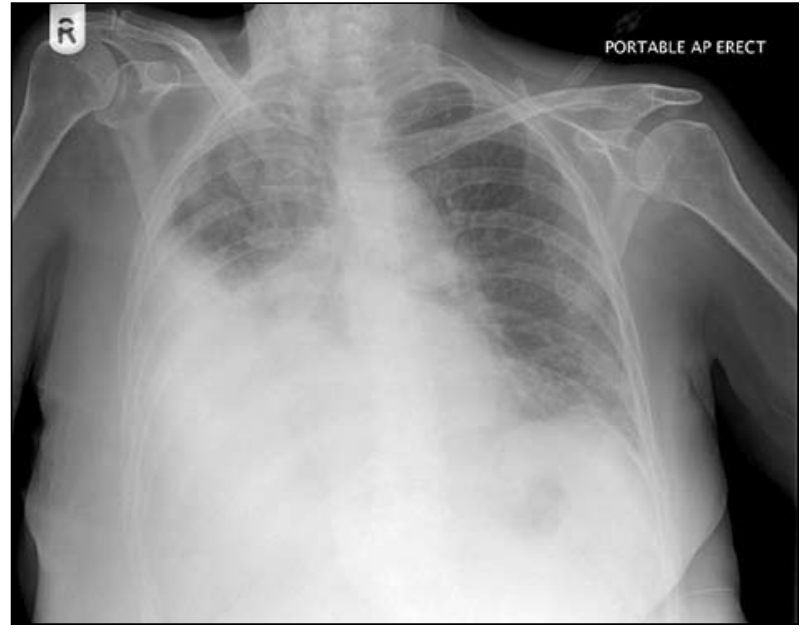

FIGURE I Chest radiograph suggestive of moderate right pleural effusion.

treated with palliative chemotherapy. She died of respiratory failure within three months of the diagnosis.

\section{DISCUSSION}

Pleural fluid analysis can provide valuable information towards the diagnosis and determining the aetiology of pleural effusion. Amylase-rich pleural effusion (ARPE) is defined as either a pleural amylase greater than the upper limit of serum amylase or a pleural fluid to serum amylase ratio of more than I. The prevalence of ARPE has been reported between $5 \%$ and $15 \%$ in different studies. $^{1-3}$ Amylase-rich pleural effusion is seen in a number of conditions, for example pancreatitis, oesophageal rupture, malignancy, pneumonia, ectopic pregnancy and liver cirrhosis, and occasionally following upper abdominal surgery. 


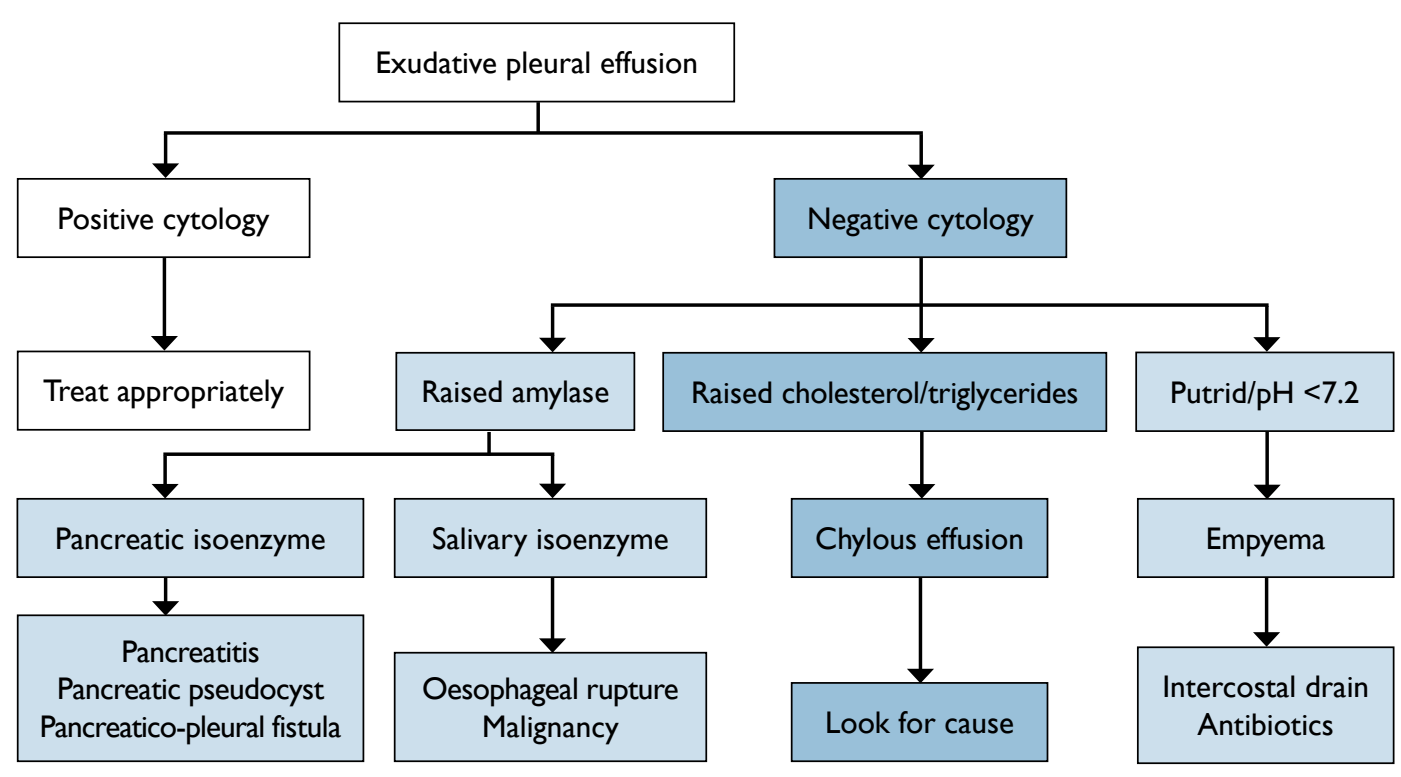

FIGURE 2 Schematic diagram for evaluating exudative pleural effusion.

According to British Thoracic Society (BTS) guidelines, ${ }^{4}$ pleural fluid amylase should be measured if acute pancreatitis or oesophageal rupture is suspected. However, isoenzyme anlaysis is useful in the evaluation of exudative effusion if there is no evidence of oesophageal perforation, as salivary amylase is raised in the cases of malignancy and pancreatic amylase is increased in pancreatic disease (Figure 2).

However, normal pleural fluid amylase does not exclude malignancy as a cause of effusion. In a prospective study of 200 patients with pleural effusions, Joseph and co-workers analysed 25 ARPEs. ${ }^{5}$ Four patients had evidence of pancreatitis, with elevated pancreatic isoenzyme in all of them. Lung malignancy (eight patients, including two adenocarcinomas) was the most common cause of non-pancreatitis ARPE, with elevated salivary isoenzyme in the majority of patients.

In a review of 841 cases of pleural effusions, Villena and colleagues found that approximately $8 \%$ of effusions were amylase rich. $^{3}$ Lung malignancy was the most common neoplastic cause of these effusions.

\section{REFERENCES}

I Ende N. Studies of amylase activity in pleural effusions and ascites. Cancer 1960; I3:283-7. doi:I0.1002/1097-0|42(I96003/04) I3:2 <283::AID-CNCR2820I302I2>3.0.CO;2-2

2 Light RW. Pleural effusions. Med Clin North Am 1977; 61:1339-52.

3 Villena V, Pérez V, Pozo F et al. Amylase levels in pleural effusions: a consecutive unselected series of 841 patients. Chest 2002; I21:470-4. doi:10.1378/chest. 121.2 .470

4 Maskell NA, Butland RJ; Pleural Diseases Group, Standards of Care Committee, British Thoracic Society. BTS guidelines for the investigation of a unilateral pleural effusion in adults. Thorax 2003; 58(Supp 2):ii8-17. doi:I0.II36/thorax.58.suppl_2.ii8
Adenocarcinoma was the most frequent histologic type ( 18 cases). Moreover, patients with pleural amylase $>600$ international units per litre (iu/l) had significantly shorter median survival.

The most likely explanation of the pathogenesis of elevated amylase in malignant effusions is the ectopic production of amylase by cancer cells, as supported by a case report of adenocarcinoma with ARPE by Kitazawa and colleagues. ${ }^{6}$ The ectopic production of amylase by malignant cells was confirmed by enzyme-labelled antibody method on pleural fluid.

\section{CONCLUSION}

This case highlights the significance of considering malignancy in conjunction with markedly raised pleural fluid amylase. Moreover, ARPE can provide useful information for evaluation of the likely aetiology, especially when cytology is negative. In addition, isoenzyme analysis can further narrow down the differential diagnosis and help identify the exact aetiology earlier, and aid in the management of exudative pleural effusions.
5 Joseph J,Viney S, Beck $\mathrm{P}$ et al. A prospective study of amylase-rich pleural effusions with special reference to amylase isoenzyme analysis. Chest 1992; 102: I455-9. doi:10.1378/chest. I02.5.1455

6 Kitazawa M, Nakagawa M, Baba $O$ et al. [A case of amylase producing lung cancer.] Kokyu To Junkan 1993; 4I:393-6. In Japanese. 